\title{
Estudio de la influencia de la presión en la oxidación de $\mathrm{H}_{2}$
}

\author{
Juan Manuel Colom, Eduardo Royo, Ángela Millera, Rafael Bilbao, María U. Alzueta \\ Grupo de Procesos Termoquímicos (GPT) \\ Instituto de Investigación en Ingeniería de Aragón (I3A) \\ Universidadde Zaragoza, Mariano Esquillor s/n, 50018, Zaragoza, Spain. \\ Tel. +34-976762707, e-mail:juanmcd@unizar.es
}

\begin{abstract}
El presente trabajo tiene como objetivo el estudio experimental de la oxidación de hidrógeno $\left(\mathrm{H}_{2}\right)$ en una instalación a presión en el intervalo de temperaturas de $300-700{ }^{\circ} \mathrm{C}$ y a presiones de 10,20 y 40 bar. Los resultados han sido simulados con el programa informático Chemkin-PRO usando un mecanismo detallado de reacción.
\end{abstract}

\section{Introducción}

El hidrógeno ha cobrado importancia en las últimas décadas debido a su potencial como energía renovable. $\mathrm{Su}$ alto contenido energético por unidad de masa y su casi inexistente contribución a la generación de contaminación lo hacen una atractiva opción, aunque el problema de su almacenamiento parece un tema pendiente [1]. Por otro lado, los esfuerzos se centran cada vez más en el desarrollo de sistemas de combustión a alta presión con el objetivo de aumentar su eficiencia. Esta tendencia crea la necesidad de probar la validez de los modelos cinéticos en dichas condiciones. Además, el sub-mecanismo $\mathrm{H}_{2} / \mathrm{O}_{2}$ es la base en los modelos cinéticos para la combustión de hidrocarburos. En los últimos años se han publicado diferentes estudios sobre la oxidación de hidrógeno [2,3], pero son pocos los que se centran en la influencia de la presión [4]. En este contexto, el presente trabajo aborda el estudio de la oxidación de hidrógeno a alta presión y diferentes temperaturas.

\section{Metodología experimental}

Los experimentos se llevaron a cabo en un reactor a presión de flujo laminar, el cual está descrito en detalle en [5]. Brevemente, la instalación consiste en un sistema de alimentación de gases, un sistema de reacción y un sistema de análisis de gases. Botellas a presión con $\mathrm{N}_{2}, \mathrm{O}_{2}$ al $1.8 \%$ e $\mathrm{H}_{2}$ al $1 \%$ suministran los gases a la instalación, introduciendo un caudal total al reactor de $1 \mathrm{~L}(\mathrm{STP}) / \mathrm{min}$. Se eligieron condiciones oxidantes, con concentraciones de 1000 ppm de $\mathrm{H}_{2}$ y 3000 ppm de $\mathrm{O}_{2}$, condiciones de interés en procesos reales de combustión. Los reactantes están altamente diluidos en nitrógeno y sus caudales se regulan mediante controladores de flujo másico. El sistema de reacción consiste en un reactor de cuarzo (diámetro interno de $6 \mathrm{~mm}$ y $1500 \mathrm{~mm}$ de longitud), en el interior de una coraza de acero inoxidable cuya función es mantener la alta presión para evitar que la paredes del reactor se rompan. Este sistema se calienta mediante resistencias entre $300{ }^{\circ} \mathrm{C}$ y $700{ }^{\circ} \mathrm{C}$ para estudiar la reacción. El hidrógeno se analiza a la salida con un cromatógrafo de gases equipado con detectores de conductividad térmica.

\section{Modelo}

Los resultados experimentales se analizaron utilizando el programa informático Chemkin-Pro, con modelo de reactor flujo pistón, y un mecanismo detallado de reacción para la conversión de hidrógeno a alta presión tomado de bibliografía [4].

\section{Resultados}

Los resultados de consumo de hidrógeno con respecto a la temperatura de reacción a las distintas presiones estudiadas se muestran en la Figura 1. Se observa que la conversión de hidrógeno se inicia a menor temperatura a medida que aumenta la presión, y que, en general, los perfiles de concentración de hidrógeno están desplazados a menores temperaturas a medida que la presión es mayor. Además, se observa una mayor influencia en el paso de 10 a 20 bar que aumentando a presión de 20 a 40 bar. La Figura 1 muestra también los resultados de simulación obtenidos con el modelo anteriormente descrito. Se observa, en general, un buen acuerdo entre los resultados experimentales y del modelo, si bien a las mayores temperaturas estudiadas el modelo sobrepredice la concentración de hidrógeno.

\section{Conclusiones y trabajo futuro}

Este trabajo muestra un estudio de la oxidación de hidrógeno a diferentes presiones $(10,20$ y 40 bar) en condiciones oxidantes. Los resultados muestran un apreciable efecto de la presión de operación, de modo que los perfiles de conversión de hidrógeno se 
desplazan a menores temperaturas a medida que aumenta la presión, siendo este efecto más importante para las menores presiones estudiadas. La simulación realizada con un mecanismo de reacción $\mathrm{H}_{2} / \mathrm{O}_{2}$ con Chemkin-PRO, ajusta bien en general los resultados de los experimentos E1 estudio será ampliado en el futuro a condiciones reductoras y estequiométricas.

\section{REFERENCIAS}

[1]. SCHLAPBACH, L., and ZÜTTEL, A. Hydrogenstorage materials for mobile applications. Nature. 2001,414(6861), 353-358.

[2]. BURKE, M.P., CHAOS, M., JU, Y., DRYER, F.L., and KLIPPENSTEIN, S.J. Comprehensive $\mathrm{H}_{2} / \mathrm{O}_{2}$ kinetic model for high-pressure combustion. International Journal of Chemical Kinetics. 2012,44(7), 444-474.
[3]. HONG, Z., DAVIDSON, D.F., and HANSON, R.K. An improved $\mathrm{H}_{2} / \mathrm{O}_{2}$ mechanism based on recent shock tube/laser absorption measurements. Combustion and Flame. 2011, 158(4), 633-644.

[4]. HASHEMI, H., CHRISTENSEN, J.M., GERSEN, S., and GLARBORG, P. Hydrogen oxidation at high pressure and intermediate temperatures: Experiments and kinetic modeling. Proceedings of the Combustion Institute. 2015, 35(1), 553-560.

[5]. MARRODÁN, L., MILLERA, A., BILBAO, R., and AlZUETA, M.U. High-Pressure study of methyl formate oxidation and its iinteraction with $\mathrm{NO}$. Energy \& Fuels. 2014, 28(9), 6107-6115.

[6]. BROMLY, J.H., BARNES, F.J., NELSON, P.F., and HAYNES, B.S. Kinetics and modeling of the $\mathrm{H}_{2}-\mathrm{O}_{2-}$ $\mathrm{NO}_{\mathrm{x}}$ system. International Journal of Chemical Kinetics. 1995, 27(12), 1165-1178.

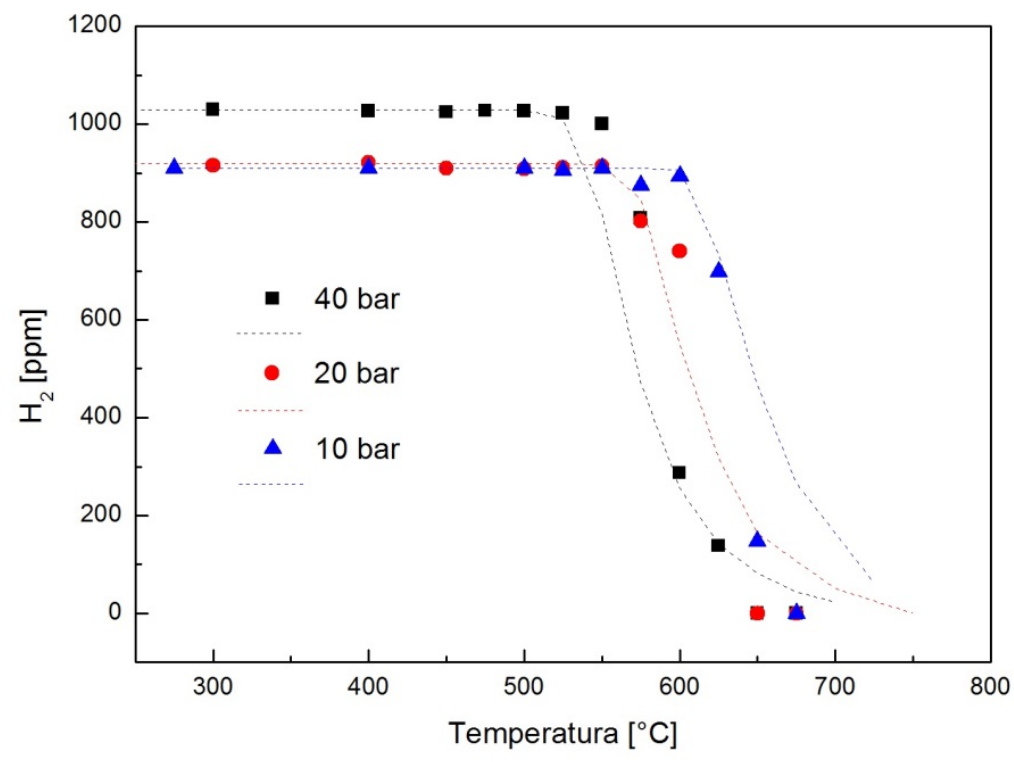

Figura 1: Concentración de hidrógeno frente a la temperatura a distintas presiones. Los símbolos respresentan resultados experimentales y las líneas resultados de simulación. 